\title{
Light Meter for Measuring Photosynthetically Active Radiation
}

\author{
Alexander Kutschera ${ }^{1}$, Jacob J. Lamb ${ }^{2 *}$ \\ ${ }^{1}$ TUM School of Life Sciences Weihenstephan, Technical University of Munich, Freising-Weihenstephan, Germany \\ ${ }^{2}$ Department of Electronic Systems \& ENERSENSE, NTNU, Trondheim, Norway \\ Email: *jacob.j.lamb@ntnu.no
}

How to cite this paper: Kutschera, A. and Lamb, J.J. (2018) Light Meter for Measuring Photosynthetically Active Radiation. American Journal of Plant Sciences, 9, 2420-2428.

https://doi.org/10.4236/ajps.2018.912175

Received: October 22, 2018

Accepted: November 16, 2018

Published: November 19, 2018

Copyright $\odot 2018$ by authors and Scientific Research Publishing Inc. This work is licensed under the Creative Commons Attribution International License (CC BY 4.0).

http://creativecommons.org/licenses/by/4.0/ Open Access

\begin{abstract}
Measurement of photosynthetically active radiation (PAR) incident on photosynthetic organisms is a crucial measurement for understanding how organisms respond to various light conditions, and for calculating electron flow through the photosynthetic machinery. Measurements of PAR are typically performed in the region of the electromagnetic spectrum between $400-700$ $\mathrm{nm}$, which is the region of radiation that is responsible for promoting photosynthesis. Typically, to ensure that the sensor measures in this range, the implementation of long- and short-pass filters is required. Although this allows the exclusion of radiation outside of the PAR region, such filters can be expensive. Additionally, the implementation of autonomous PAR measurements requires costly commercial instruments. Here, a straight-forward, inexpensive apparatus has been designed and constructed using a sensor that can distinguish between red, green, blue and white light. The constructed apparatus was able to perform comparably to a commercial PAR sensor. Furthermore, the implementation of the device to measure PAR intensity over a three-day period shows how the apparatus can be implemented for use as a constant light monitor.
\end{abstract}

\section{Keywords}

Photosynthesis, Light Meter, Photosynthetically Active Radiation

\section{Introduction}

Methods for accurately determining PAR are required continuously within the research field of photosynthesis and oceanography, where the determination of the incident light in a given area is measured in $\mu \mathrm{mol}$ per square meter per 
second. PAR is defined as the radiation that is available for photosynthesis [1] [2]. The wavelength range is determined by light absorption capacity of the pigments located in the photosynthetic machinery, which are involved in the light-dependent reactions of photosynthesis. Here, solar energy is converted into chemical energy through several chained redox reactions for use by the organism [3]. This, in turn, is used for $\mathrm{CO}_{2}$ fixation during the light-independent reactions of photosynthesis [4].

Due to the absorption spectra of the photosynthetic pigments, the central electromagnetic radiation wavelengths of interest fall between $400-700 \mathrm{~nm}$ [3]. These are the wavelengths that are utilised by the photosynthetic machinery of the organism [1]. This means that solar energy available for the plant metabolism can be determined by measuring the PAR at the location of the organism [2]. In turn, this information can be used to estimate the yield at sites of interest or in scientific studies analysing variances in PAR utilisation between different photosynthetic species [5]. Globally, PAR is used as a proxy for carbon fixation by photosynthesis and has a significant impact on the transport of water from the surface of the earth, into the atmosphere [6] [7]. Therefore, determination of PAR is also crucial to calculating the carbon balance in climate research [5] [6] [7].

As stated, PAR is usually measured as $\mu$ mol photons $\mathrm{m}^{-2} \mathrm{~s}^{-1}$ using sensors with wavelength specific filters (400 $\mathrm{nm}$ long-pass filter and a $700 \mathrm{~nm}$ short-pass filter); however, these components can be expensive. Thus, an ideal sensor should be able to measure radiation between $400-700 \mathrm{~nm}$ very efficiently while excluding other, photosynthetically inactive, radiation. Many commercially available sensors are close to an ideal sensor and are already widely in use for the applications stated above. However, commonly used quantum sensors are expensive making wide-spread use of large area monitoring infeasible by the majority of research institutes and companies [8] [9]. The open source science hardware movement has led to the development of low-cost versions of scientific instruments with performance comparable to commercial products [10]. While there are multifunctional devices available [11], there is also a need for low-cost stand-alone PAR sensors that can be deployed in large numbers to monitor PAR over a large area for an extended period.

This report describes how to construct a microcontroller-based modular PAR sensor, highlighting the performance parameters, and demonstrating its application. The apparent level of PAR in a given light setting is determined using the described instrument in correlation to a commercially available light sensor set up to measure PAR.

\section{Materials and Methods}

\section{Materials and Instrumentation of Custom-Build Light Meter}

An Arduino UNO microcontroller board was used to power the photodiode, and to digitise the detected PAR light using the integrated 8-bit, high precision 
analogue to digital converter (ADC). The internal FLASH memory of the Arduino was programmed to control the data acquisition, and processing using the Arduino integrated development environment (arduino.cc). The measuring system can operate autonomously using an LCD screen display (an HD44780-driver-compatible, $5 \mathrm{~V}, 20 \times 2$ Character LCD), and battery.

Black PLA (Polylactic acid) plastic was used by a 3D printer (Ultimaker Original+, Ultimaker, The Netherlands) to construct the sensor form that housed the photodiode (measuring unit). A diffuse light barrier covers the centre of the sensor unit. The amount of light was detected by a photodiode (TCS34715FN, AMS-TAOS Inc.) positioned on the sensor form. The photodiode was powered (pin 1 on the photodiode) by the $3.3 \mathrm{~V}$ power output pin of an Arduino. The inter-integrated circuit $\left(\mathrm{I}^{2} \mathrm{C}\right)$ communication used two bidirectional open-drain ports; the serial data line (SDL/SDA) port, and the serial clock line (SCL) port. Pin 2 on the photodiode connected to the SCL port on the Arduino, and was pulled up by also connecting the SCL port to the $3.3 \mathrm{~V}$ power output through a $10 \mathrm{~K} \Omega$ resistor. Pin 6 on the photodiode was connected to the SDL port on the Arduino and was also pulled up in the same manner as the SCL port. The photodiode was grounded through pin 3.

\section{Comparison of Light Sensors}

The constructed light sensor was compared to a commercial LI-COR 190R quantum sensor in a variety of light environments. To compare the two instruments, both the constructed instrument and the commercial instrument were placed side-by-side in a variety of locations in the lab and office, and outside during clear and cloudy sunlight. The results from both sensors was obtained from each instrument and recorded manually.

Continual Light Measurement over 72 hours

To measure the incident light, the sensor unit was placed on a flat area in direct sunlight on the roof of a building. The placement took into account the potential for surrounding objects to cast a shadow upon the sensor, and an area unaffected by shadowing from structures was chosen. The Arduino board was connected to a laptop computer to log the data during the experiment.

\section{Results}

The determination of PAR for photosynthetic applications is of importance as an analytical parameter when measuring photosynthetic activity. PAR typically spans the wavelength range of $400-700 \mathrm{~nm}$ and measured in units of $\mu \mathrm{mol}$ photons $\mathrm{m}^{-2} \mathrm{~s}^{-1}$. This light range is the range actively used in many photosynthetic organisms. Although expensive light filters have been required in traditional instruments for excluding light outside the PAR range, the use of an off-the-shelf RGBW sensor has proved to remove the requirement of these filters. By using an algorithm developed by Kuhlgert et al. (2016), PAR light values can be approximated using the outputs of the four light channels obtained from the photodiode [11]. 
We have constructed an instrument that can obtain PAR values, which utilises a photodiode to determine the incident light. This report details the performance of the instrument, as well as providing the technical details to allow researchers with a limited background in electronics to construct such a light sensor.

\section{Microcontroller Choice}

The use of small mixed-signal microcontrollers facilitates the design of scientific instruments. For this modular instrument, the Arduino Uno mixed-signal microcontroller was used for powering the photodiode, as well as storing and digitising the data. The microcontroller chip (ATmega328P) has an ADC successive-approximation-register on-chip. This offers a real, linear, 8-bit accuracy that is satisfactory for the requirements of the modular photometer. The Arduino also has SCL and SDL/SDA ports for $\mathrm{I}^{2} \mathrm{C}$ communication, necessary for the specific photodiode used.

\section{Photodiode Choice}

A TCS34715FN photodiode was chosen for use in the modular photometer. It is an inexpensive photodiode that can measure white light, blue light, red light and green light independently. It also responds to light in the range of $300-1100$ $\mathrm{nm}$ (with a linear dependence between the PAR region of $400-700 \mathrm{~nm}$ on the white light channel). The benefit of the onboard operational amplifier is the reduction of potential noise as the signal is amplified before leaving the photodiode chip, reducing the likelihood of interference.

\section{Design}

The light sensor setup enables simple construction and use of the measuring system. The base module consists of a microcontroller and one measurement unit. A schematic for the measuring setup is presented in Figure 1.

\section{Microcontroller Interfacing}

The schematic representation in Figure 2 displays the interfacing of the microcontroller with the external components (photodiode and LCD screen). The chosen Arduino microcontroller has various input and output ports. The photodiodes pin 1 was connected to the $3.3 \mathrm{~V}$ power output pin of an Arduino, pin 2 to the SCL port of the Arduino, pin 3 to a ground port of the Arduino and pin 6

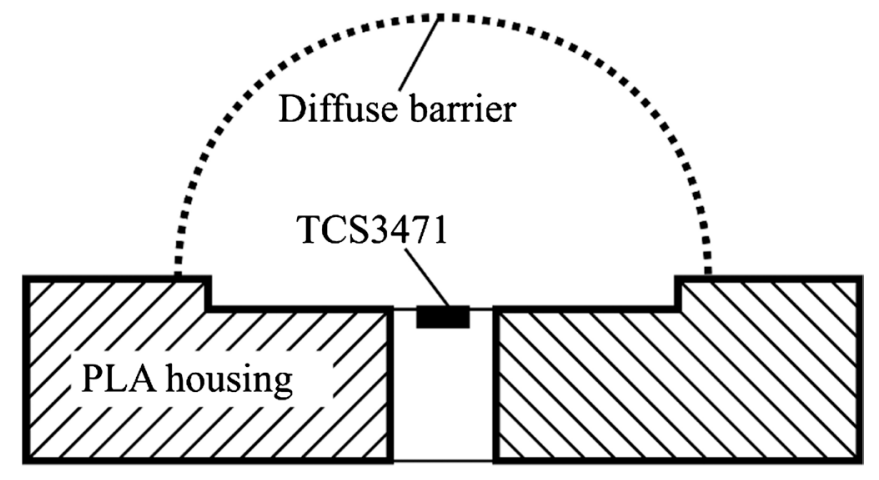

Figure 1. Sensor module. Cross-section of the sensor module constructed from 3D-printed black PLA plastic, a half hemisphere white diffuser, and a photodiode. 


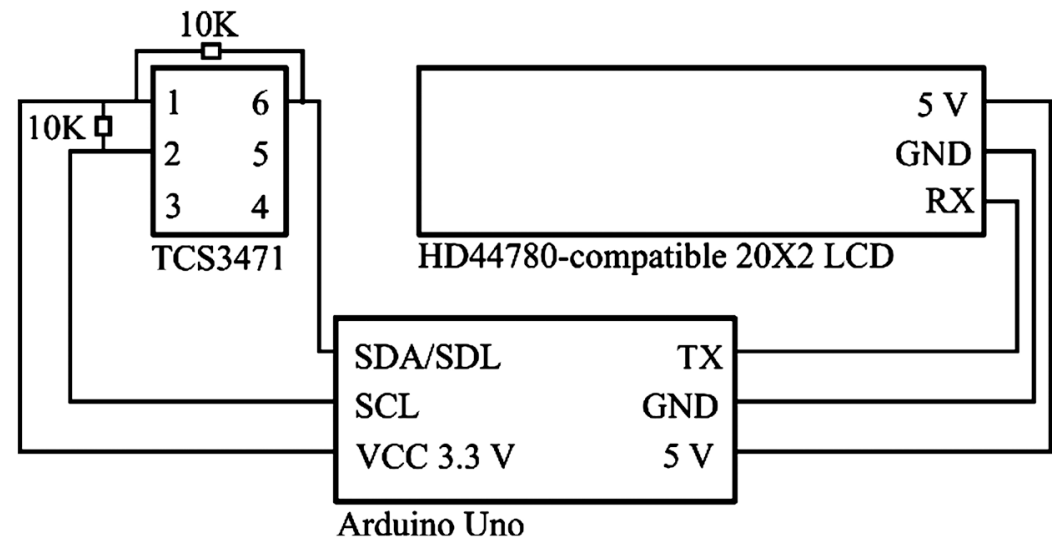

Figure 2. Wiring schematic. The layout of the photodiode, microcontroller and LCD display showing they are connected to each other.

to the SDA port of the Arduino. Both the SDA and SCL ports on the Arduino we connected individually to the $3.3 \mathrm{~V}$ pin of the Arduino through $10 \mathrm{~K} \Omega$ resistors.

The LCD screen can be powered directly from the $5 \mathrm{~V}$ pin on the microcontroller, and grounded by connecting to a ground pin on the microcontroller. The RX pin from the LCD screen connects directly to the TX pin (pin 1) of the microcontroller to transmit the data to be displayed. Monitoring can also be achieved using a PC or single-board computer. The microcontroller connects to the Raspberry Pi single-board computer through a USB cable, allowing the live monitoring of the output of the module via serial communication.

\section{Microcontroller Algorithm and Software}

Upon power-up, the microcontroller will start the measuring script and will send the live data through the serial port to the LCD screen, and a PC if connected. Additional updates of the firmware installed on the microcontroller can be performed in this connected mode. As described by Kuhlgert et al. (2016), measurements from the TCS34715FN photodiode were corrected using coefficients derived using a pseudo-inverse matrix equation in comparison to the PAR values obtained from an LI-COR LI-190R PAR sensor [11]:

$$
\begin{aligned}
\text { PAR }= & (\text { White } \times 0.65847)+(\text { Red } \times 1.60537) \\
& +(\text { Green } \times 2.30216)+(\text { Blue } \times 0.50019)
\end{aligned}
$$

This results in an incident PAR reading in $\mu$ mol photons $\mathrm{m}^{-2} \mathrm{~s}^{-1}$ that can be used directly for a variety of applications.

\section{Measurement of PAR}

To characterise the performance of the PAR sensor different light qualities were used. These included natural sunlight (with and without cloud cover) and various artificial white light sources. The performance of the modular light meter is comparable to that of the commercial light sensor used (LI-COR 190R) when measuring the natural PAR (Figure 3). A small deviation between the PAR measured may be apparent between the two instruments as the PAR value increases, but this is assumed to be due to the different arrangements of optical components in the instruments used. 

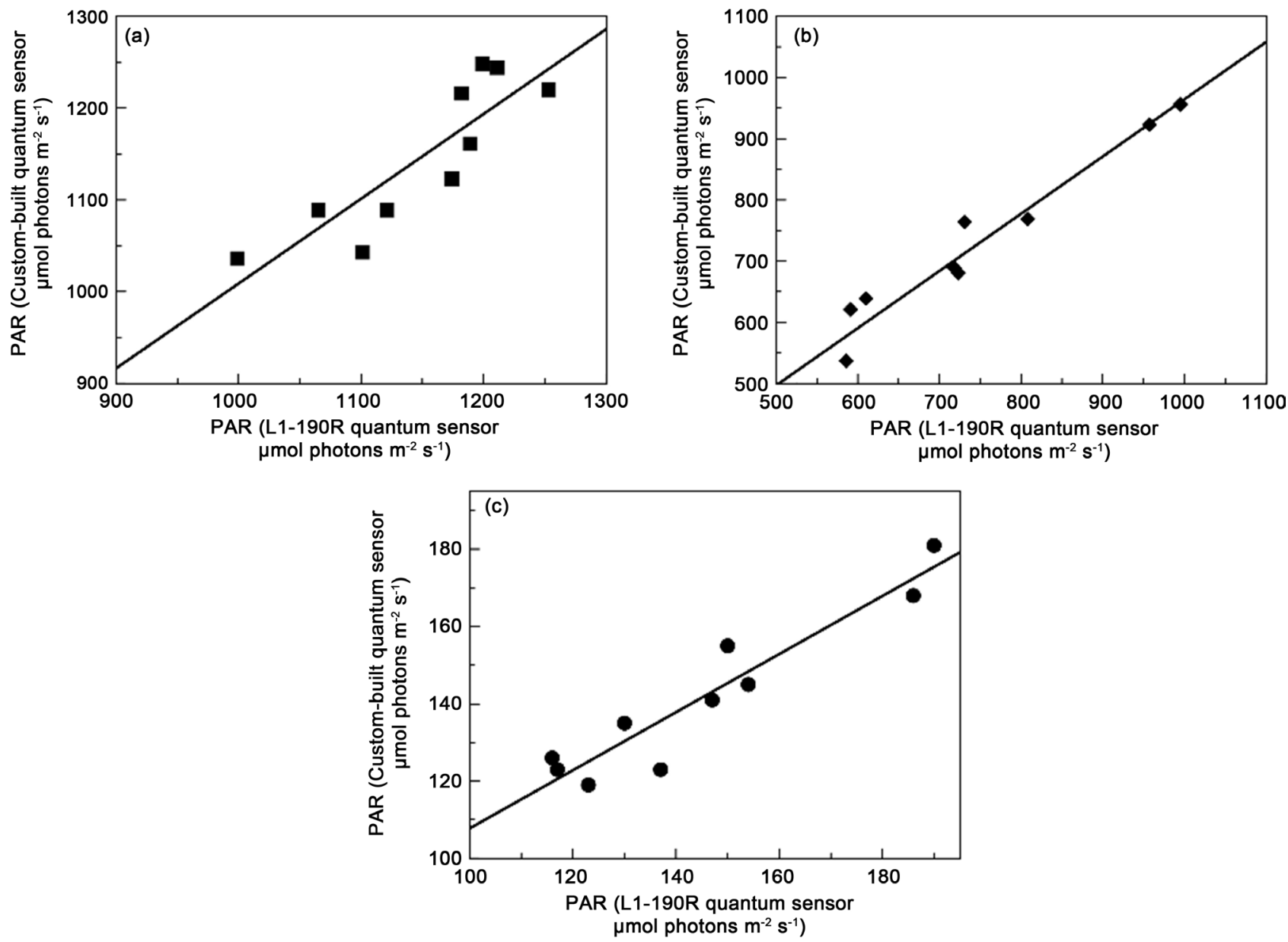

Figure 3. Comparison to commercial quantum PAR sensor. The custom-built PAR sensor was compared to a LI-COR 190R commercial quantum sensor. The environments tested were during (a) a cloudless sunny day $(\mathrm{y}=0.9231 \mathrm{x}+85.896)$; (b) during a cloudy day $(y=0.9348 x+29.636)$; and $(c)$ indoors with various white light sources $(y=0.5801 x+58.086)$.

\section{Live Monitoring of PAR}

To evaluate the live monitoring performance of the PAR sensor, the sensor of the constructed instrument was placed outside the laboratory window for three days. The PAR was monitored continuously for 72 hours at a frequency of one measurement every 20 minutes. The live performance has been shown in Figure 4. During this 72-h period, there was high-level partial cloud cover, with patches of clear sky on the first day. As the experiment progressed, the cloud height reduced, causing the clouds to affect the incident light on the sensor. This is shown in Figure 4, with a trend of reduced maximal light sensed as the experiment progressed into day two and three.

\section{Discussion}

This report has described and shown the construction of a live PAR monitor that can precisely determine the PAR of natural and artificial light. The measurement of PAR recognises the incident light on a surface between $400-700 \mathrm{~nm}$. The PAR can change proportionally to the amount of light incident on a given surface. 


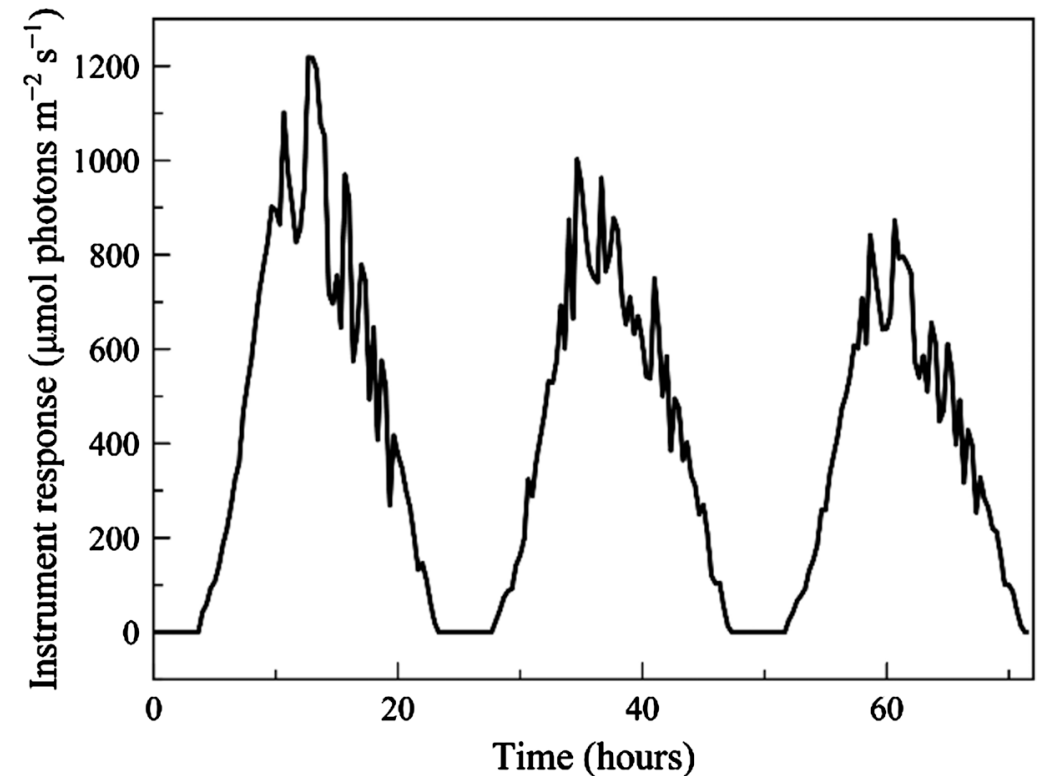

Figure 4. Live monitoring of PAR. PAR monitored outdoors over a 72-hour period in Trondheim, Norway (late April 2018), with partial dynamic cloud cover. Measurements were taken autonomously every 20 minutes throughout the 72 hours.

Despite the strong correlation between the PAR values observed in the constructed apparatus, and the commercial light sensor, small deviations may appear.

This modular PAR sensor is based on a 3D-printed module, and an inexpensive, widely obtainable microcontroller platform for powering the LCD, and a photodiode. The microcontroller also converted the photodiodes analogue signal into a digital signal and communicated this data to a PC for live data streaming. The PAR sensor has a low cost of around USD 40 - 50 to build and might be reduced even further when it is used as a data logger without an LCD, making it an economically appealing option for many research groups wanting live measurements of PAR.

The constructed instrument was compared to a commercial LI-COR 190R quantum sensor. The comparison was made in a variety of indoor and outdoor conditions (cloudless sunny day, cloudy day and indoors with various white light sources in the lab and the office). In the comparison performed, a close linear relationship between the commercial instrument, and the constructed instrument was observed. This suggested that the constructed instrument provides similar PAR measurements to a commercial alternative, and is further able to perform continual measurements over a time period of $72 \mathrm{~h}$. It is assumed that this time period can be significantly increased without significant differences in performance.

The Arduino compiling software is free and has been made available for Microsoft Windows, Linux, and MacOS platforms. The design of the Arduino boards facilitates the use and design of electronic instruments by non-experts. Furthermore, the ease of firmware designing and programming allows greater 
flexibility for the end-user to tailor the modular photometer to meet their specific experimental requirements. This can all be achieved using a standard PC with a USB port.

An advantage of this instrument is its simplicity. Although it is possible to obtain a variation in the amount of light detected between different instruments dependent on how the components of the instrument are arranged, the PAR sensor uses mass-produced components that are identical, and if used in the same arrangement, reproducible results from multiple PAR sensors is achievable. This allows the live monitoring of multiple light sources simultaneously. The PAR sensor allows online real-time plotting and analysis of the light measurements. This may facilitate monitoring natural or artificial light conditions for photosynthetic research.

\section{Conclusion}

In summary, the design, construction, characterisation, and calibration of a live PAR sensor for determining the incident light intensity between $400-700 \mathrm{~nm}$ have been presented. It provides an easy-to-use, modular, cost-effective, and reliable solution to light intensity measurements, allowing the live monitoring a light environment. The presented instrument will help many laboratories produce simultaneous live measurements without a sizeable financial requirement.

\section{Acknowledgements}

Jacob Lamb acknowledges the support from the ENERSENSE research initiative, and his research was supported by a post-doctoral fellowship from the Norwegian University of Science and Technology-NTNU.

\section{Conflicts of Interest}

The authors declare that they have no conflict of interest.

\section{References}

[1] McCree, K.J. (1971) The Action Spectrum, Absorptance and Quantum Yield of Photosynthesis in Crop Plants. Agricultural Meteorology, 9, 191-216. https://doi.org/10.1016/0002-1571(71)90022-7

[2] McCree, K.J. (1972) Test of Current Definitions of Photosynthetically Active Radiation against Leaf Photosynthesis Data. Agricultural Meteorology, 10, 443-453. https://doi.org/10.1016/0002-1571(72)90045-3

[3] Lodish, H., Berk, A., Zipursky, S.L., Matsudaira, P., Baltimore, D. and Darnell, J. (2000) Photosynthetic Stages and Light-Absorbing Pigments. Molecular Cell Biology. 4th Edition, W. H. Freeman, New York.

[4] Black Jr, C.C. (1973) Photosynthetic Carbon Fixation in Relation to $\mathrm{Net}_{\mathrm{CO}_{2}} \mathrm{Up}$ take. Annual Review of Plant Physiology, 24, 253-286. https://doi.org/10.1146/annurev.pp.24.060173.001345

[5] Gitelson, A.A., Peng, Y., Arkebauer, T.J. and Suyker, A.E. (2015) Productivity, Absorbed Photosynthetically Active Radiation, and Light Use Efficiency in Crops: Im- 
plications for Remote Sensing of Crop Primary Production. Journal of Plant Physiology, 177, 100-109. https://doi.org/10.1016/j.jplph.2014.12.015

[6] Bondeau, A., Smith, P.C., Zaehle, S., Schaphoff, S., Lucht, W., Cramer, W., et al. (2007) Modelling the Role of Agriculture for the 20th Century Global Terrestrial Carbon Balance. Global Change Biology, 13, 679-706.

https://doi.org/10.1111/j.1365-2486.2006.01305.x

[7] Frouin, R. and Pinker, R.T. (1995) Estimating Photosynthetically Active Radiation (PAR) at the Earth's Surface from Satellite Observations. Remote Sensing of Environment, 51, 98-107. https://doi.org/10.1016/0034-4257(94)00068-X

[8] Akitsu, T., Nasahara, K.N., Hirose, Y., Ijima, O. and Kume, A. (2017) Quantum Sensors for Accurate and Stable Long-Term Photosynthetically Active Radiation Observations. Agricultural and Forest Meteorology, 237, 171-183.

https://doi.org/10.1016/j.agrformet.2017.01.011

[9] Barnes, C., Tibbitts, T., Sager, J., Deitzer, G., Bubenheim, D., Koerner, G., et al. (1993) Accuracy of Quantum Sensors Measuring Yield Photon Flux and Photosynthetic Photon Flux. HortScience, 28, 1197-1200.

[10] Murillo, L.F., Molloy, J. and Dosemagen, S. (2018) Global Open Science Hardware Roadmap.

[11] Kuhlgert, S., Austic, G., Zegarac, R., Osei-Bonsu, I., Hoh, D., Chilvers, M.I., et al. (2016) MultispeQ Beta: A Tool for Large-Scale Plant Phenotyping Connected to the Open PhotosynQ Network. Royal Society Open Science, 3, 160592.

https://doi.org/10.1098/rsos.160592 